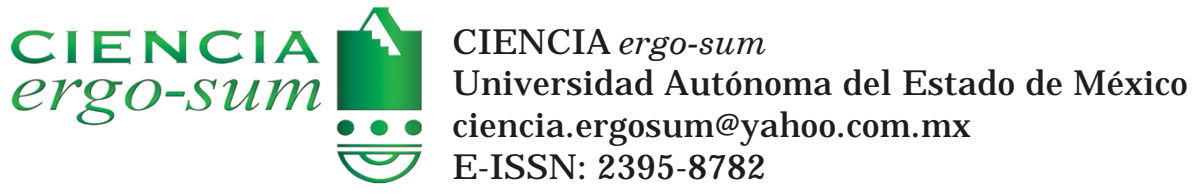

\title{
Participación en el mercado de crédito formal versus el informal en México
}

Guizar, I saí; González Olivares, Daniel; Ezzahra Housni, Fatima

Participación en el mercado de crédito formal versus el informal en México

CIENCIA ergo-sum, vol. 27, núm. 2, julio-octubre 2020|e81

Universidad Autónoma del Estado de México, México

Esta obra está bajo una Licencia Creative Commons Atribución-NoComercial-SinDerivar 4.0 Internacional.

Guizar, I ., González Olivares, D. y Ezzahra Housni, F. (2020). Participación en el mercado de crédito formal versus el informal en México. CIENCIA ergo-sum, 27(2). https://doi.org/10.30878/ces.v27n2a2 


\title{
Participación en el mercado de crédito formal versus el informal en México
}

Participation in Formal versus Informal Credit Markets in Mexico

\author{
Isai Guizar \\ Universidad de Guadalajara, México \\ isai.guizar@cucea.udg.mx \\ (D) https://orcid.org/0000-0001-8616-8848 \\ Daniel González Olivares \\ Universidad de Guadalajara, México \\ daniel.gonzalez.o@cucea.udg.mx \\ (D) https://orcid.org/0000-0002-9052-2763 \\ Fatima Ezzahra Housni \\ Universidad de Guadalajara, México \\ fatima.housni@cusur.udg.mx \\ (1) https://orcid.org/0000-0003-0660-609X
}

\author{
Recepción: 4 de julio de 2018 \\ Aprobación: 30 de octubre de 2019
}

\section{RESUMEN}

Se identifican factores que influyen en la decisión de tomar un crédito formal versus uno informal en México. Empleando información de la Encuesta Nacional de Inclusión Financiera, los resultados econométricos distinguen un conjunto de variables que tienen una relación positiva en las elecciones de fuentes formales, pero negativa en las informales (ingreso, educación, estado civil, género y acceso a seguros), otra que sólo influye en las elecciones formales (posesión de garantías, tamaño del hogar, ahorro financiero y posesión de teléfono celular) y otra en las elecciones de mecanismos informales (edad). El ejercicio ayuda a comprender por qué la participación de los mexicanos en el mercado de crédito formal es substancialmente menor que en el mercado informal.

Palabras ClaVe: elecciones de crédito, inclusión financiera, servicios financieros.

\begin{abstract}
This paper identifies factors that influence the decisions to take credits from formal and from informal sources in Mexico. Employing data from the Mexican National Survey of Financial Inclusion, the econometric results reveal a set of factors that have a positive effect on the choices of formal credit, but negative in the choice of informal sources (income, education, marital status, gender, and access to insurance), another set that statistically explains only the choice of formal sources (ownership of collateral, size of the household, financial savings, and ownership of cellphones), and a variable that only influences the use of informal sources (age). The study helps to improve our understanding of why participation in the formal sector in Mexico remains low relative to its informal counterpart.
\end{abstract}

KEYWORDS: Credit Choices, Financial Inclusion, Financial Services.

\section{INTRODUCCIÓN}

Si bien la reasignación de los recursos en la economía por parte de agentes con exceso de fondos (prestamistas) hacia agentes que sufren de escasez (prestatarios) permite que se acelere la producción y el crecimiento (Levine, 1997, 2005; Levine et al., 2000), en la práctica existen problemas de información asimétrica, de incompatibilidad de incentivos, fallas institucionales y costos de transacción (Jafee y Stiglitz, 1990; Besley, 1994; Conning y Udri, 2007; Giné, 2011) que frecuentemente obstruyen su movilización a través de canales formales.

Como resultado de estas imperfecciones del mercado emergen las restricciones de oferta de servicios financieros (Jafee y Stiglitz, 1990) que, en función de su severidad, llegan a inhibir por completo la presencia del mercado 
formal y excluyen a poblaciones de muy bajos ingresos en primera instancia porque son quienes típicamente enfrentan mayores costos de transacción (González-Vega, 2003) y carecen de los requisitos elementales para que el prestamista evalúe su solvencia como historiales de crédito y garantías líquidas (Demirgüç-Kunt et al., 2008).

Las dificultades para medir la magnitud de estas restricciones, a su vez, impiden evaluar con precisión sus posibles impactos en el acceso y uso de crédito formal. Para México se presumen condiciones severas, pues, tomando como referencia los indicadores de Inclusión Financiera Global del Banco Mundial (2018a), únicamente 10.4\% de los mexicanos obtuvo un préstamo de una institución financiera formal en 2014 , mientras que $26.0 \%$ obtuvo crédito de familiares o amigos, esto es, aproximadamente 2.5 veces más informal que formal. Para 2017 los reportes de la misma fuente indican que fue $5.7 \%$ el que obtuvo préstamos de fuentes formales y $14.0 \%$ de familiares o amigos; sin entrar aquí en debates sobre metodologías de medición, basta resaltar que las cifras muestran que la relación de participación en los sectores formal e informal se ha mantenido, i. e., las barreras a la movilización de fondos en el sector formal parecieran persistentes.

Fuentes informales como familiares, prestamistas locales o casas de empeño sustituyen, por lo menos parcialmente, la escasez y restricciones de acceso al crédito formal. En diferentes contextos se ha encontrado que, a comparación del crédito formal, el informal es típicamente más rápido de obtener, genera menores costos de transacción, es más racionado o riesgoso, en tanto que frecuentemente se ofrece a tasas de interés también más elevadas (Mohieldin y Wright, 2000; Giné, 2011).

Ahora bien, dada las restricciones del crédito formal, el informal es necesario, pero el resultado económico no es el óptimo. La literatura también documenta efectos positivos de acceso a crédito formal a poblaciones excluidas en educación, inversión, creación de negocios, acumulación de capital humano, estabilización del consumo y adopción de tecnologías de producción de mayor rentabilidad (Lloyd-Ellis y Bernhardt, 2000; McKenzie y Woodruff, 2008; Maldonado y Gonzalez-Vega, 2008; Banerjee et al., 2015).

Reconociendo entonces la importancia del crédito formal en la economía y los rezagos en la materia que sufre México, el propósito de este trabajo es documentar los factores que determinan la elección entre el crédito formal e informal de los mexicanos. Los resultados obtenidos aportarán información que facilite la compresión del funcionamiento de la demanda de crédito y, de esta forma, el diseño de políticas de inclusión crediticia.

En la siguiente sección se muestra un panorama general de uso de mecanismos de crédito formal versus informal en el contexto internacional, la dos analiza el contexto mexicano, en la tres se describe el ejercicio econométrico y las conclusiones generales se presentan en la última sección.

\section{Participación en los mercados de crédito formal e informal en el contexto} INTERNACIONAL

Al describir la participación en el mercado de crédito de los países que presentamos a continuación enfatizamos el rol del nivel de ingreso. Como notaremos posteriormente, es uno de los principales determinantes para elegir el tipo crédito. La gráfica 1 muestra el empleo de crédito formal (ofertado por instituciones financieras) en diferentes países, a la izquierda para miembros de la Organización para la Cooperación y Desarrollo Económico (OCDE) y a la derecha sólo para países de Latinoamérica (LA). Destacan varios aspectos: a) En ningún país latinoamericano la proporción de encuestados que empleó un crédito formal supera 50\%, mientras que en los países de la OCDE cerca de la mitad de los países reportan el empleo del crédito de una institución formal en una proporción mayor a 50\%.b) El ingreso per cápita más alto en los países de Latinoamérica lo tuvo Chile con cerca de 15 000. En el grupo de miembros de la OCDE, del que Chile también forma parte, solamente cinco países (incluyendo México) tienen menores ingresos menores a esa cifra. c) La relación entre ingresos y empleo de crédito formal es positiva y clara en ambos grupos de países. Los coeficientes de correlación son de 0.63 y 0.76 para el grupo de la OCDE y de Latinoamérica respectivamente. 

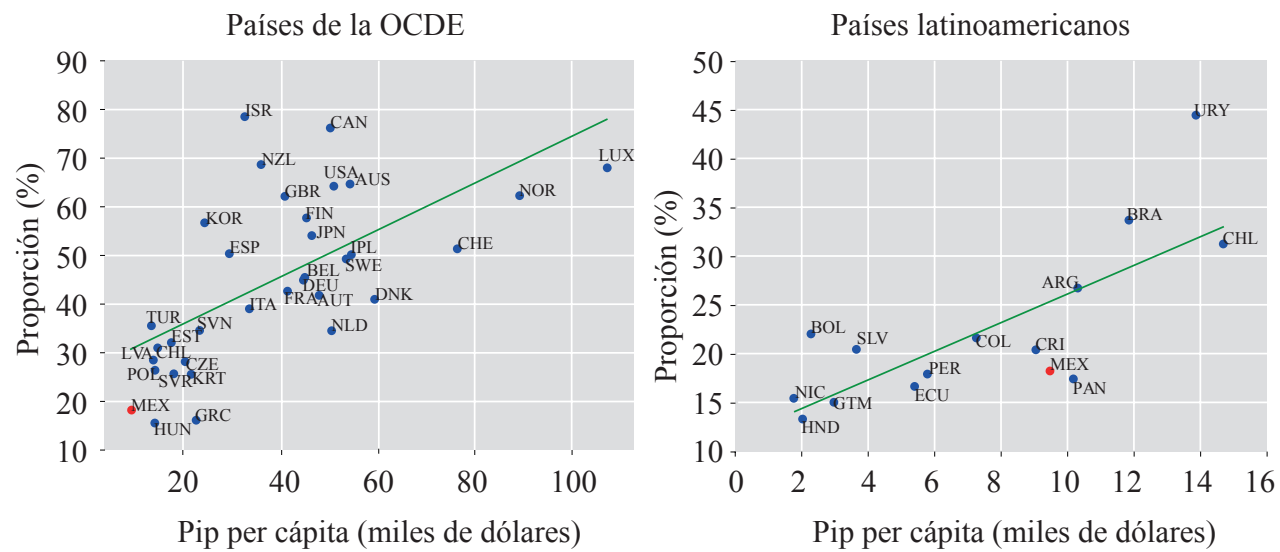

GRÁFICA 1

Participación en el mercado de crédito formal (2014)

Fuente: elaboración propia con datos de los Indicadores de Desarrollo Mundial y de la Inclusión Financiero Global Banco Mundial (2018b; 2018a).

La relación del ingreso con uso de crédito informal se muestra en la gráfica 2 y se utiliza la proporción de personas que tuvieron un préstamo de familiares o amigos para aproximar crédito informal. En general, en ambos grupos de países se percibe una relación negativa. En este caso los coeficientes de correlación son de -0.37 y -0.35 para el grupo OCDE y Latinoamérica respectivamente. Las gráficas de este artículo muestran que en los países de mayores ingresos el uso de mecanismos formales de crédito es mayor y entre menor es el ingreso mayor es el uso de mecanismos informales.
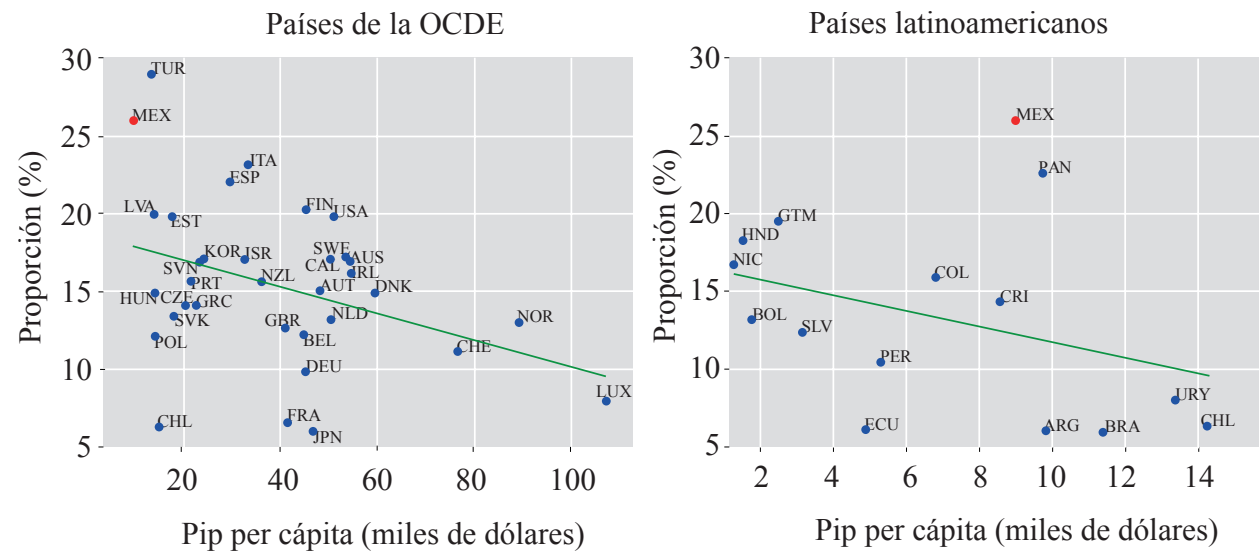

GRÁFICA 2

Participación en el mercado de crédito informal (2014)

Fuente: elaboración propia con datos de los Indicadores de Desarrollo Mundial y del

Desarrollo Financiero Global Banco Mundial (2018b) .

México es el país de menor ingreso (USD 9 493) y también el de menor uso de crédito formal (18.28\%) en la OCDE (gráfica 1). En el grupo de países latinoamericanos el ingreso de México es cercano al promedio; no obstante, forma con Perú, Panamá, Ecuador, Guatemala, Nicaragua y Honduras el subgrupo que menos utiliza mecanismos formales de crédito. De forma clara, tanto en países de la OCDE como en los de Latinoamérica, México está por debajo de la línea de tendencia en cuanto al empleo de préstamos de una institución formal (gráfica 1) y muy por encima de las líneas de ajuste en lo que respecta al empleo de mecanismos informales (gráfica 2), es decir, la formalidad (informalidad) en el uso de crédito en México es atípicamente baja (alta) respecto a países con niveles de ingreso similar. 


\section{Participación en los mercados de crédito formal e informal en México}

Para analizar el uso de crédito en México se emplea la Encuesta Nacional de Inclusión Financiera (ENIF) de 2015. La ENIF es representativa a nivel nacional, y reporta información de 6039 viviendas que pertenecen a 252 municipios de la República Mexicana. Es ideal porque permite distinguir la participación de los individuos en el mercado formal del informal, así como los mecanismos y motivos por los que se emplean.

\section{1. Mercado formal}

Quienes reportan tener algún tipo de crédito formal, conforman 29\% (1 752 entrevistados) y $71 \%$ que no lo tiene representa a 4287 entrevistados, de ellos 867 alguna vez lo tuvieron y 3420 nunca han participado en el mercado de crédito formal. Conviene examinar a los tres grupos por separado: a) del grupo que sí tiene un crédito interesa conocer qué mecanismos utilizan y para qué lo disponen, $b$ ) de los que alguna vez tuvieron se indagan los motivos por los que dejaron de participar y $c$ ) de los que nunca han participado se busca conocer las razones de su exclusión.

En el primer grupo se encuentra que las tarjetas de crédito, ya sea departamentales/tiendas de autoservicio (66.7\%) o bancarias (36.9\%) son los mecanismos más comunes para adquirir el crédito formal (gráfica 3). El uso de otros mecanismos no excede 20\%; en orden descendente son los hipotecarios (15.5\%), personales (13.2\%), de nómina (9.1\%), grupales (8.6\%) y el automotriz (4.6\%).
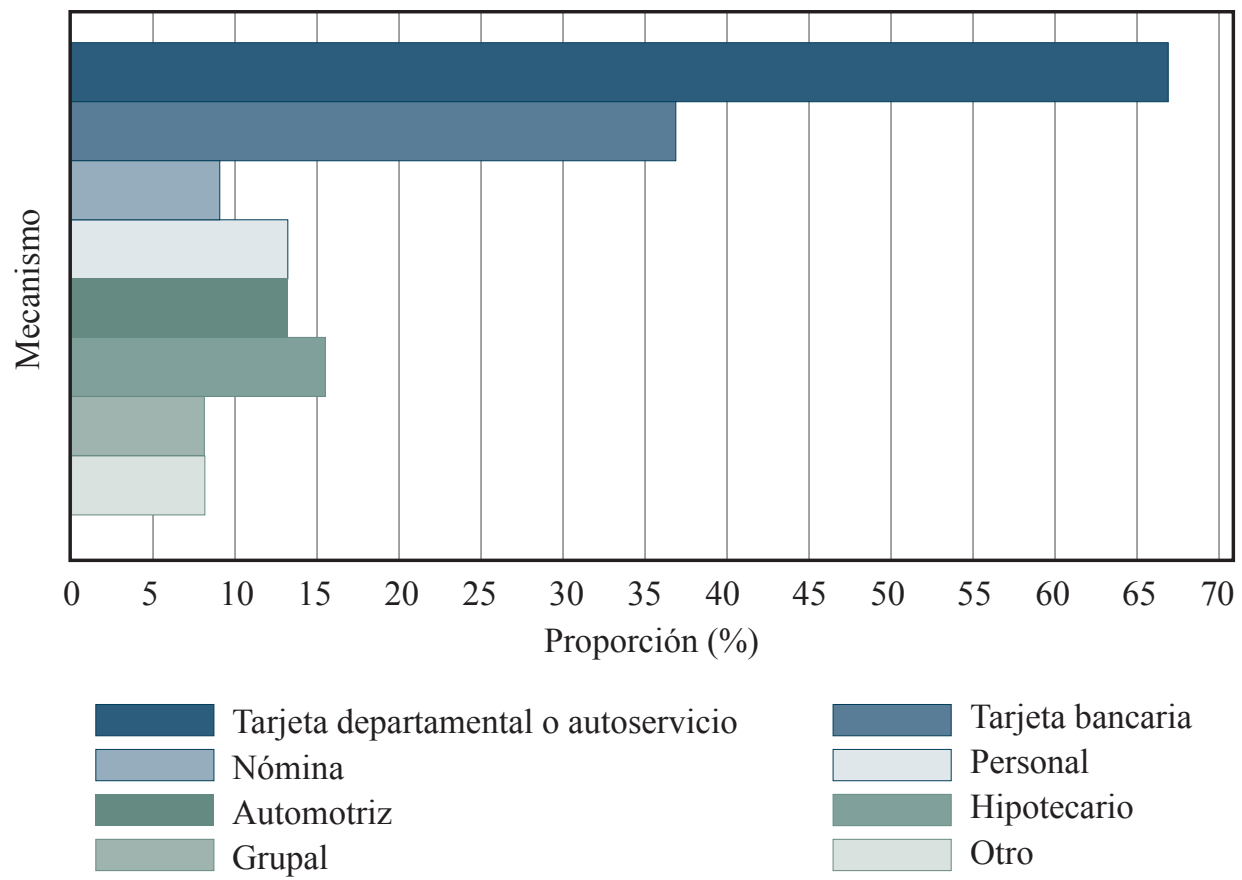

\section{GRÁFICA 3}

Tipos de crédito formal utilizados

Fuente: elaboración propia con datos de la ENIF, 2015.

Del total de entrevistados que posee algún préstamo, $82.4 \%$ lo obtiene a través de una tarjeta de crédito. La tabla 1 resume las respuestas reportadas en cuanto a su uso y señala que las tarjetas de crédito se usan principalmente para compras de establecimientos comerciales (66.4\%). Le siguen los retiros en efectivo de cajeros automáticos $(14.8 \%)$ para pago de servicios (13.9\%) y menor proporción para gastos en el negocio $(5.8 \%)$. 
TABLA 1

Destino del crédito obtenido a través de tarjetas

\begin{tabular}{lc} 
Destino & Porcentaje \\
\hline Retiros en cajeros automáticos & 14.8 \\
Compras en establecimientos comerciales, tiendas, etc. & 66.4 \\
Pago de servicios & 13.9 \\
Gastos del negocio & 5.8 \\
\hline
\end{tabular}

Fuente: elaboración propia con datos de la ENIF, 2015.

En cuanto al uso de crédito no predestinado que conforman los préstamos personales, de nómina y los grupales, la tabla 2 muestra que la mayor parte de los mexicanos lo utiliza para la adquisición o restauración de activos como terrenos, vehículos o joyas (29.7\%). El segundo uso preferido es la compra de alimentos, gastos personales y pago de servicios $(24.9 \%)$, le sigue con $11.8 \%$ un préstamo para pagar otra deuda y $6.4 \%$ con fines vacacionales o festivos.

TABLA 2

Destino del crédito personal, de nómina o grupal

\begin{tabular}{|cc} 
Destino & Porcentaje \\
Gastos de alimentación, personales o pago de servicios & 24.9 \\
Gastos de salud & 8.3 \\
Gastos de educación & 8.8 \\
Atender una emergencia o imprevistos & 8.0 \\
Compra o restauración de activos & 29.7 \\
Pagar una deuda & 11.8 \\
Comenzar, ampliar u operar un negocio & 16.4 \\
Vacaciones o fiesta & 6.4 \\
Otro & 3.6
\end{tabular}

Fuente: elaboración propia con datos de la ENIF, 2015.

En el segundo grupo conformado por los 837 entrevistados que dejaron de participar en el mercado de crédito formal, la tabla 3 identifica que la razón principal es el elevado interés en el préstamo (28.7\%), después la falta de voluntad para contraer otra deuda (25.6\%), la falta necesidad de solicitar un préstamo (24.8\%) y las malas experiencias con las instituciones financieras (19.8\%). Las razones que contiene la categoría de Otro, mencionadas po $15 \%$ de los usuarios, refieren primordialmente a factores de riesgo en el ingreso. Entonces, excluyendo el conjunto que no necesita un nuevo préstamo, se logra apreciar que son las condiciones financieras y el trato que reciben las personas por parte de la institución como las razones principales de abandono.

En la tabla 4 se enlistan los motivos por los que el tercer grupo integrado por 3420 individuos nunca ha participado en el mercado formal. Excluyendo a los que no necesitan o no quieren contraer deuda, se encuentra la incapacidad de cumplir con los requisitos solicitados (34.0\%), lo elevados costos de interés/comisiones (13.7\%), la desconfianza en las instituciones financieras (4.9\%) y la lejanía a la sucursal (1.8\%).

No se debe omitir que $33.9 \%$ de la población que nunca ha tenido un crédito formal sí participa en el mercado informal; esto es coherente con los estudios que señalan que el crédito informal funciona como un sustituto parcial a la falta de accedo al formal (Diagne, 1999; Mohieldin y Wright, 2000). Las razones de exclusión mencionadas por los entrevistados permiten señalar que en México, en la formulación de políticas que fomenten la participación 
en el mercado crediticio formal, se deberá reconocer que una mayor inclusión financiera requiere de esfuerzos que promuevan la creación de infraestructuras de comunicación más efectivas, que reduzcan las distancias, que promuevan la confianza de las personas en instituciones financieras y que fomenten la competencia entre instituciones crediticias que permita reducir los intereses y comisiones que el usuario absorbe.

TABLA 3

Motivos por el que dejó de participar en el mercado formal de crédito

\begin{tabular}{|lc|}
\hline Razón & Porcentaje \\
\hline Intereses muy altos & 28.7 \\
No se quiere volver a endeudar & 25.6 \\
No lo necesita & 24.8 \\
Mala experiencias con instituciones financieras & 19.8 \\
No cumple con los requisitos & 3.6 \\
Prefiere crédito informal & 1.4 \\
Otro & 15.0 \\
\hline
\end{tabular}

Fuente: elaboración propia con datos de la ENIF, 2015.

TABLA 4

Motivos por el que no ha participado en el mercado formal de crédito

\section{Razón}

No le gusta endeudarse

No cumple con los requisitos

No lo interesa/necesita

Altos intereses o comisiones

Desconfianza en las instituciones financieras

Cree que lo van a rechazar

Larga distancia a la sucursal

Otro

\section{Porcentaje}

39.5

34.0

18.4

13.7

4.9

2.1

1.8

6.8

Fuente: elaboración propia con datos de la ENIF, 2015.

\section{2. Mercado informal}

En el mercado informal participa 38.6\% de la población. El principal financiador es la familia y amigos (29.3\%) y para cerca de la mitad de los entrevistados que acuden a ellos es su única fuente de crédito, es decir, no fueron expulsados del sector formal, sino que nunca han pertenecido. Las casas de empeño (7.8) y cajas de ahorro del trabajo y conocidos (7.1) también cumplen un rol de prestamistas informales, pero son menos utilizadas (gráfica 4).

La mayor proporción de usuarios de crédito informal destina el crédito a gastos de alimentación, personales o de servicios. Nótese que mientras $25.9 \%$ de los usuarios formales le daba el mismo uso, la proporción aquí es de 34.1. En el rubro de gastos de salud, en el informal es $22 \%$, mientras que en el formal sólo 8.3\%. De forma contraria, en el informal $12.8 \%$ reporta usarlo para adquirir activos y $6.3 \%$ para el negocio, mientras que en el formal el uso es 29.7 y $16.4 \%$ respectivamente. Se puede percibir que el crédito informal se destina en mayor medida a necesidades básicas como alimentación, salud y educación, en tanto que el formal a gastos secundarios como creación de activos, negocios o vacaciones (tabla 5). 


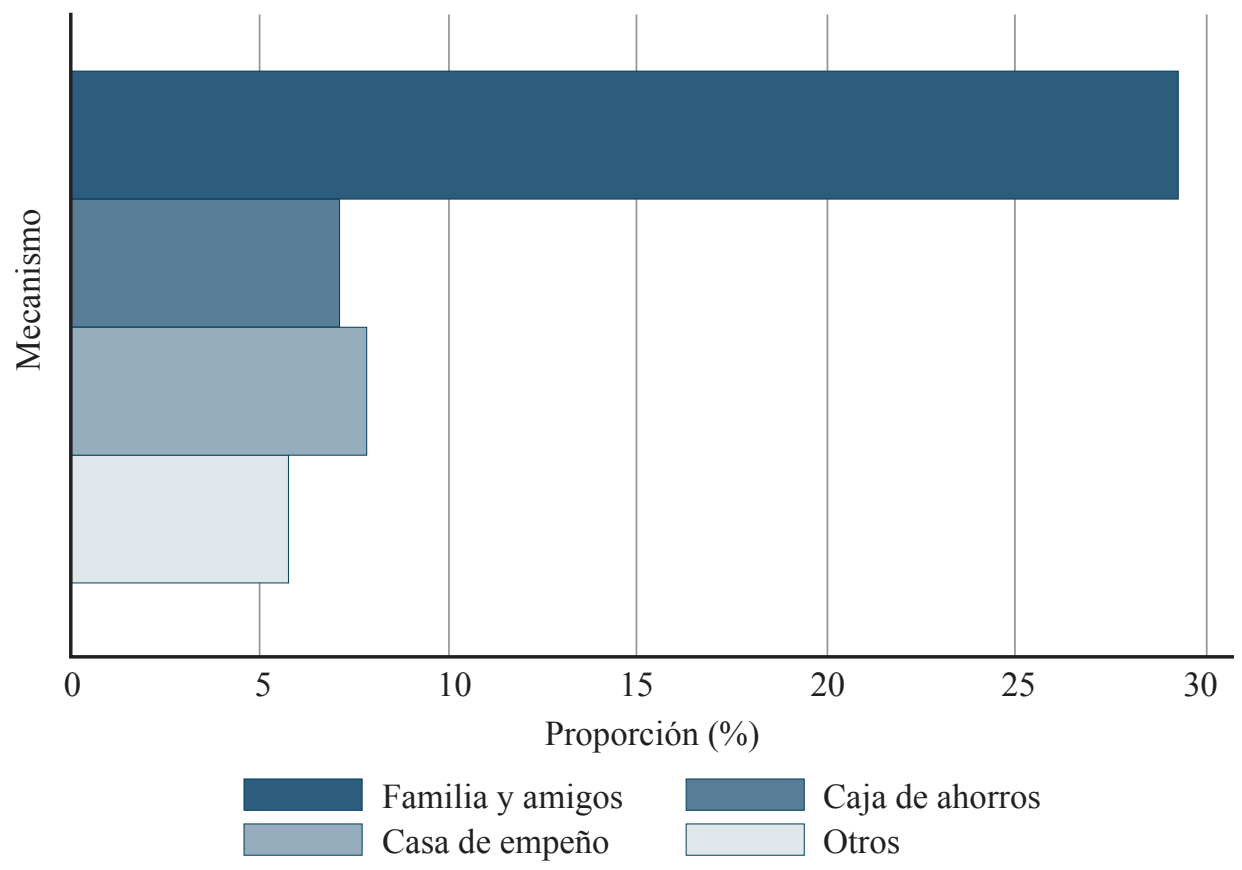

GRÁFICA 4

Tipos de crédito informal utilizados

Fuente: elaboración propia con datos de la ENIF, 2015.

TABLA 5

Destino del crédito informal

\section{Destino}

Porcentaje

Gastos de alimentación, personales o pago de servicios

35.1

Gastos de salud

22.0

Gastos de educación

Atender una emergencia o imprevistos

Compra o restauración de activos

Pagar una deuda

Comenzar, ampliar u operar un negocio

Vacaciones o fiesta

Otro

Fuente: elaboración propia con datos de la ENIF, 2015.

Para concluir esta sección indagamos si, como en los datos de países en el contexto internacional, la prevalencia del tipo de crédito en las distintas entidades del país se mantiene asociada a sus niveles de ingreso. El mapa 1 a) y b) ilustra la proporción de usuarios de crédito formal (izquierda) y la de usuarios de crédito informal (derecha) y el c) muestra el nivel promedio de ingreso. La intensidad del color representa el nivel de la variable: entre más oscuro, mayor es el valor.

Las cinco entidades con mayor penetración de crédito formal son Baja California Sur, Nayarit, Sinaloa, Sonora y Yucatán, donde la proporción de usuarios de crédito formal supera 40\%. Las cinco donde el crédito formal es menos empleado son Chiapas, Michoacán, Tlaxcala, Guerrero y Puebla, donde la proporción es menor a 20\%. Por otra parte, el crédito informal prevalece en Guerrero, Yucatán, Sinaloa, Quintana Roo y Aguascalientes con 
una participación mayor a 45\%; en el otro extremo están el Distrito Federal, Zacatecas, Tamaulipas y Chiapas, donde el empleo de mecanismos informales es inferior a 33\%. Merece la pena mencionar que Guerrero es el único estado que aparece en la lista de estados con menor crédito formal y de mayor informal.

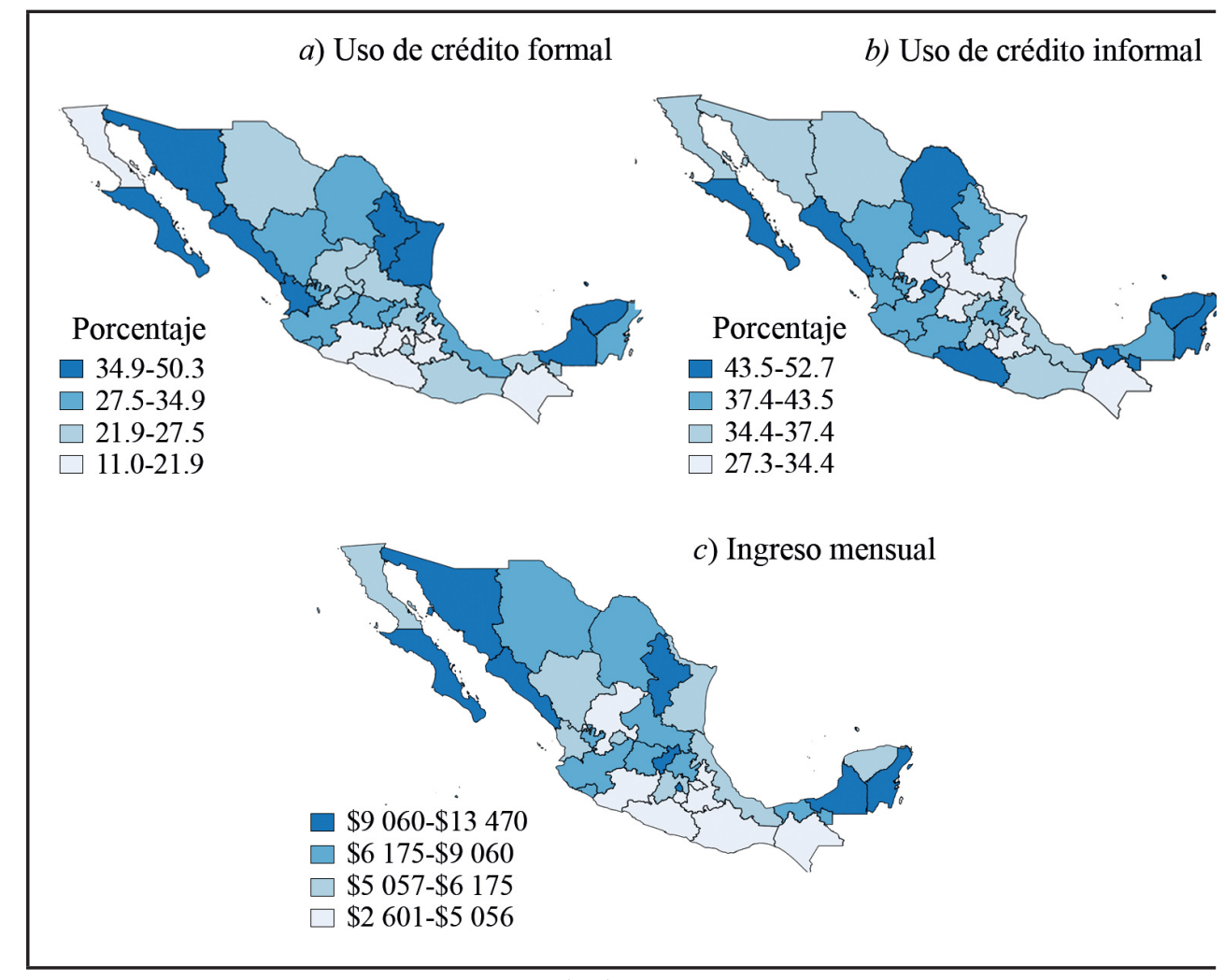

MAPA 1

Participación estatal en el mercado de crédito formal, informal e ingreso mensual Fuente: elaboración propia con datos de la ENIF, 2015.

En el mapa c) aparece el nivel promedio de ingreso mensual que reportaron los entrevistados. Los de menor ingreso son algunos estados del Sureste y Centro, destacan Guerrero, Michoacán, Chiapas y Puebla con ingresos inferiores a los $\$ 4000$; en tanto que las entidades que reportan los ingresos mas altos son Baja California Sur, Querétaro, Sonora, Ciudad de México (entonces Distrito Federal) y Quintana Roo con ingresos superiores a los $\$ 9500$. La correlación entre ingresos y uso de crédito formal es de 0.64 , positiva y estadísticamente significativa. Con el informal es también positiva, pero sólo 0.14 y no significativa a niveles de confianza convencionales.

\section{EJERCICIO ECONOMÉTRICO}

El objetivo del ejercicio econométrico será identificar variables que ayuden a explicar las preferencias por los distintos tipos de crédito de los mexicanos. Los datos de la ENIF son apropiados para realizar un análisis de corte transversal. La variable dependiente representa la elección de crédito, interpretada como una variable latente. El modelo se especifica como:

$$
\text { crédito* }=\alpha_{0}+X \alpha_{1}+\epsilon
$$

donde la variable indicadora crédito $=1$ si el individuo participa en el mercado de crédito en cuestión $($ crédito* $<0$ ) y 0 si no participa. La matriz $X$ contiene el conjunto de variables explicativas y $\epsilon$ es el término de error, donde se asume que $X$ es independiente de $\epsilon$ y que la distribución acumulada del término de error es $F$ : 


$$
P[\text { crédito }=1 \mid X]=P\left[\epsilon>-\left(\alpha_{0}+X \alpha_{1}\right) \mid X\right]
$$

que es el modelo clásico de probabilidad:

$$
P[\text { crédito }=1 \mid X]=F\left(\alpha_{0}+X \alpha_{1}\right)
$$

La definición de las variables dependientes que representan crédito se realiza considerando que el interés principal del artículo es analizar los grupos que eligen exclusivamente crédito formal o informal. Del universo de entrevistados, 13\% participa simultáneamente en los dos mercados de crédito, 45\% no participa en ninguno, 16\% es exclusivamente formal y $26 \%$ exclusivamente informal (cuadro 1). Entonces, omitimos al grupo que participa en ambos, de tal forma que se realizan dos conjuntos de estimaciones: en el primero la variable dependiente toma valores de 1 si el individuo participa sólo en el formal y 0 si no participa, en tanto que en el segundo conjunto la dependiente toma el valor de 1 si el individuo participa exclusivamente en el informal y 0 si no participa en el mercado. Cada conjunto incluye tres diferentes especificaciones: la estimación del modelo lineal que sirve como referencia y posteriormente los modelos no lineales con la función de distribución del error normal (probit) y logística (logit).

\section{CUADRO 1}

Distribución de la participación en los mercados de crédito

\begin{tabular}{lcccc|} 
& No participa & Formal & Informal & Ambos \\
\hline Número & 2735 & 969 & 1552 & 783 \\
Porcentaje & $45 \%$ & $16 \%$ & $26 \%$ & $13 \%$ \\
\hline
\end{tabular}

Fuente: elaboración propia.

Los resultados de las estimaciones se presentan en el cuadro 2. Se muestra que el ingreso, el nivel educativo, ser mujer jefa de familia, estado civil y la característica de poseer algún tipo de seguro (como proxy para acceso a otros servicios financieros) tienen una relación estadísticamente significativa con la elección del crédito. El efecto de todas ellas es positivo en la elección de crédito formal, pero negativo en la elección de crédito informal. Como se había previsto en la descripción de las secciones anteriores, a mayor nivel de ingreso mayor es la probabilidad de que el individuo posea un crédito formal y reduce la probabilidad de que seleccione un mecanismo informal. Tampoco sorprende que el nivel educativo impacte de forma positiva la inclusión en el mercado formal y la negativa a las elecciones informales pues mayor educación permite un mejor entendimiento de los productos financieros y reduce los costos de transacción.

Los resultados también muestran que es más probable que las mujeres prefieran un crédito formal y que los hombres elijan el informal. Así, el éxito de programas de inclusión estará condicionado a la medida con la que el producto financiero esté diferenciado por género. Tener un seguro sirve para capturar si los individuos en general tienen acceso a los mercados financieros. Los signos reflejan algún grado de complementariedad entre el acceso al mercado de seguros y al de crédito formal.

El efecto de poseer activos físicos como terrenos, viviendas, automóviles también es positivo en la elección de crédito formal, pero con efecto nulo en el informal. El resultado tampoco sorprende, pues el tener garantías de pago del crédito facilita el acceso a mecanismos formales, pero no es relevante en los informales. El menor tamaño del hogar, medido por el número de personas, el hecho de poseer un teléfono celular y tener una cuenta de ahorro financiero, que sirven para aproximar la capacidad de pago, también son factores relacionados con la participación en el mercado formal, pero con el informal la relación estadística es nula. Mientras que la edad 
sirve para predecir participación en el informal, en el formal el efecto estadístico es 0 . Este resultado tiene implicaciones sobre acceso a crédito considerando el ciclo de vida, un tema que deberá ser tratado con mayor profundidad en estudios complementarios, pues advierte que el ciclo de vida de la población condicionará el efecto de políticas financieras en variables macroeconómicas.

El efecto del resto de variables utilizadas, acceso a servicios médicos, número de cajeros automáticos y número de sucursales en el municipio, es estadísticamente nulo en ambos mercados.

\section{CUADRO 2}

Determinantes de participación en el mercado de crédito formal e informal

\begin{tabular}{|c|c|c|c|c|c|c|}
\hline & \multicolumn{3}{|c|}{ Crédito formal } & \multicolumn{3}{|c|}{ Crédito informal } \\
\hline & Lineal & Probit & Logit & Lineal & Probit & Logit \\
\hline Ingreso & $\begin{array}{l}0.003^{* * *} \\
(0.001)\end{array}$ & $\begin{array}{l}0.008^{* * *} \\
(0.002)\end{array}$ & $\begin{array}{l}0.003^{* * *} \\
(0.001)\end{array}$ & $\begin{array}{l}-0.001^{* * *} \\
(0.000)\end{array}$ & $\begin{array}{l}-0.005^{* *} \\
(0.002)\end{array}$ & $\begin{array}{l}-0.001^{* * *} \\
(0.000)\end{array}$ \\
\hline $\begin{array}{l}\text { Educación } \\
\text { [max. grado 0-9] }\end{array}$ & $\begin{array}{l}0.013^{* * *} \\
(0.003)\end{array}$ & $\begin{array}{l}0.050^{* * *} \\
(0.011)\end{array}$ & $\begin{array}{l}0.013^{* * *} \\
(0.003)\end{array}$ & $\begin{array}{l}-0.010^{* * *} \\
(0.003)\end{array}$ & $\begin{array}{l}-0.030^{* * *} \\
(0.010)\end{array}$ & $\begin{array}{l}-0.010^{* * *} \\
(0.003)\end{array}$ \\
\hline Sexo $[1=$ hombre $]$ & $\begin{array}{l}-0.028^{* * *} \\
(0.011)\end{array}$ & $\begin{array}{l}-0.122^{* * *} \\
(0.045)\end{array}$ & $\begin{array}{l}-0.028^{* * *} \\
(0.011)\end{array}$ & $\begin{array}{l}0.031^{* *} \\
(0.013)\end{array}$ & $\begin{array}{l}0.091^{* *} \\
(0.039)\end{array}$ & $\begin{array}{l}0.031^{* *} \\
(0.013)\end{array}$ \\
\hline Estado civil [1 = casado] & $\begin{array}{l}0.031^{* * *} \\
(0.011)\end{array}$ & $\begin{array}{l}0.138^{* * *} \\
(0.046)\end{array}$ & $\begin{array}{l}0.031^{* * *} \\
(0.011)\end{array}$ & $\begin{array}{l}-0.024^{*} \\
(0.013)\end{array}$ & $\begin{array}{l}-0.078^{*} \\
(0.040)\end{array}$ & $\begin{array}{c}-0.024^{*} \\
(0.013)\end{array}$ \\
\hline Seguro [1 = sí] & $\begin{array}{l}0.144^{* * *} \\
(0.017)\end{array}$ & $\begin{array}{l}0.460^{* * *} \\
(0.055)\end{array}$ & $\begin{array}{l}0.144^{* * *} \\
(0.017)\end{array}$ & $\begin{array}{l}-0.047^{* * *} \\
(0.017)\end{array}$ & $\begin{array}{l}-0.141^{* * *} \\
(0.053)\end{array}$ & $\begin{array}{l}-0.047^{* * *} \\
(0.017)\end{array}$ \\
\hline Activos [1 = sí $]$ & $\begin{array}{l}0.037^{* * *} \\
(0.011)\end{array}$ & $\begin{array}{l}0.146^{* * *} \\
(0.049)\end{array}$ & $\begin{array}{l}0.037^{* * *} \\
(0.011)\end{array}$ & $\begin{array}{c}0.022 \\
(0.014)\end{array}$ & $\begin{array}{c}0.064 \\
(0.042)\end{array}$ & $\begin{array}{c}0.022 \\
(0.014)\end{array}$ \\
\hline Miembros del hogar & $\begin{array}{c}-0.006^{* *} \\
(0.003)\end{array}$ & $\begin{array}{l}-0.027^{* *} \\
(0.013)\end{array}$ & $\begin{array}{c}-0.006^{* *} \\
(0.003)\end{array}$ & $\begin{array}{c}0.005 \\
(0.004)\end{array}$ & $\begin{array}{c}0.014 \\
(0.010)\end{array}$ & $\begin{array}{c}0.005 \\
(0.004)\end{array}$ \\
\hline Celular [1 = sí $]$ & $\begin{array}{l}0.071^{* * *} \\
(0.011)\end{array}$ & $\begin{array}{l}0.391^{* * *} \\
(0.060)\end{array}$ & $\begin{array}{l}0.071^{* * *} \\
(0.011)\end{array}$ & $\begin{array}{c}0.012 \\
(0.016)\end{array}$ & $\begin{array}{c}0.036 \\
(0.046)\end{array}$ & $\begin{array}{c}0.012 \\
(0.016)\end{array}$ \\
\hline Cuenta de ahorro [1 = sí $]$ & $\begin{array}{l}0.059^{* * *} \\
(0.012)\end{array}$ & $\begin{array}{l}0.262^{* * *} \\
(0.048)\end{array}$ & $\begin{array}{l}0.059^{* * *} \\
(0.012)\end{array}$ & $\begin{array}{c}0.003 \\
(0.014)\end{array}$ & $\begin{array}{c}0.011 \\
(0.042)\end{array}$ & $\begin{array}{c}0.003 \\
(0.014)\end{array}$ \\
\hline Edad & $\begin{array}{c}0.003 \\
(0.002)\end{array}$ & $\begin{array}{l}0.01 \\
(0.010)\end{array}$ & $\begin{array}{c}0.003 \\
(0.002)\end{array}$ & $\begin{array}{l}0.008^{* * *} \\
(0.003)\end{array}$ & $\begin{array}{l}0.026^{* * *} \\
(0.009)\end{array}$ & $\begin{array}{c}0.008^{* * *} \\
(0.003)\end{array}$ \\
\hline Edad*edad & $\begin{array}{c}0.000 \\
(0.000)\end{array}$ & $\begin{array}{c}0.000 \\
(0.000)\end{array}$ & $\begin{array}{c}0.000 \\
(0.000)\end{array}$ & $\begin{array}{l}-0.000^{* * *} \\
(0.000)\end{array}$ & $\begin{array}{l}-0.000^{* * *} \\
(0.000)\end{array}$ & $\begin{array}{l}-0.000^{* * *} \\
(0.000)\end{array}$ \\
\hline Servicio médico [1 = sí] & $\begin{array}{c}0.007 \\
(0.013)\end{array}$ & $\begin{array}{c}0.038 \\
(0.058)\end{array}$ & $\begin{array}{c}0.007 \\
(0.013)\end{array}$ & $\begin{array}{c}-0.014 \\
(0.017)\end{array}$ & $\begin{array}{c}-0.041 \\
(0.048)\end{array}$ & $\begin{array}{c}-0.014 \\
(0.017)\end{array}$ \\
\hline $\begin{array}{l}\text { Cajeros automáticos } \\
\text { [número] }\end{array}$ & $\begin{array}{c}0.000 \\
(0.000)\end{array}$ & $\begin{array}{c}0.000 \\
(0.000)\end{array}$ & $\begin{array}{c}0.000 \\
(0.000)\end{array}$ & $\begin{array}{c}0.000 \\
(0.000)\end{array}$ & $\begin{array}{c}0.000 \\
(0.000)\end{array}$ & $\begin{array}{c}0.000 \\
(0.000)\end{array}$ \\
\hline $\begin{array}{l}\text { Sucursales bancarias } \\
\text { [número] }\end{array}$ & $\begin{array}{c}0.000 \\
(0.000)\end{array}$ & $\begin{array}{c}0.001 \\
(0.001)\end{array}$ & $\begin{array}{c}0.000 \\
(0.000)\end{array}$ & $\begin{array}{c}0.000 \\
(0.000)\end{array}$ & $\begin{array}{c}0.001 \\
(0.001)\end{array}$ & $\begin{array}{c}0.000 \\
(0.000)\end{array}$ \\
\hline Constante & $\begin{array}{c}-0.038 \\
(0.050)\end{array}$ & $\begin{array}{c}-1.889^{* * *} \\
(0.227)\end{array}$ & $\begin{array}{c}-0.038 \\
(0.050)\end{array}$ & $\begin{array}{l}0.252^{* * *} \\
(0.064)\end{array}$ & $\begin{array}{c}-0.721^{* * *} \\
(0.191)\end{array}$ & $\begin{array}{l}0.252^{* * *} \\
(0.064)\end{array}$ \\
\hline Observaciones & 5256 & 5256 & 5256 & 5256 & 5256 & 5256 \\
\hline (pseudo) R2 & 0.13 & 0.129 & 0.13 & 0.026 & 0.023 & 0.026 \\
\hline
\end{tabular}

Notas: los errores estándar se reportan entre paréntesis. La significancia estadística indica ${ }^{*} p<0.10,{ }^{* *} p<0.05 \mathrm{y}^{* * *} p<0.01$. Estimación del modelo lineal con errores estándares robustos. Se incluyen controles de localización con ocho regiones: Noroeste, Noreste, Occidente, Oriente, Centronorte, Centrosur, Suroeste y Sureste 
Las estimaciones anteriores permiten reconocer variables que influyen en las elecciones de crédito y que por lo tanto determinan el éxito de políticas de inclusión financiera. No obstante, se debe reconocer que las relaciones encontradas no implican causalidad. Algunas variables como el ingreso pueden presentar problemas de causalidad simultánea. Factores intrínsecos al individuo como la motivación o la aversión al riesgo son variables que también pueden influir en las elecciones de crédito, pero que no son observables y, dado el tipo de datos utilizados, no fue posible incorporar en las estimaciones.

\section{Conclusiones y PRospectiva}

Con el objetivo de mejorar la compresión de por qué la participación de los mexicanos en el mercado de crédito informal es aproximadamente 2.5 veces mayor que en el formal, se examinaron datos microeconómicos de inclusión financiera en México. Los datos descriptivos revelan que, comparado con el sector formal, en el sector informal una mayor proporción de personas destina el crédito a resolver problemas de primera necesidad como alimentación y salud. El ejercicio econométrico permite identificar claramente un conjunto de variables que tienen una relación opuesta con las elecciones de los dos tipos de crédito, otro que influye sólo en las elecciones formales y otro que es únicamente significativo en las elecciones de mecanismos informales.

En el primer conjunto está el nivel de ingreso, altamente significativo con las elecciones de crédito. Los ingresos relativamente elevados incrementan la probabilidad de participar en el formal y reducen la probabilidad de participar en el informal. No obstante, el hecho de que cerca de $34 \%$ de la población que nunca ha tenido un crédito formal sí participa en el informal es indicio de que si bien menores ingresos pueden incrementar la probabilidad de rechazo de la institución financiera, no necesariamente refleja incapacidad de pago. Por ello las tecnologías crediticias que logren romper barreras de información de forma más efectiva podrán también incidir en mayor inclusión crediticia.

El mayor nivel educativo, personas del sexo femenino, de estado civil casado y poseer algún tipo de seguro también tiene una relación positiva con la contratación de un crédito formal y negativa con la del informal. Mayor educación facilita la comunicación entre oferentes y demandantes, y disminuye los costos de transacción; por lo tanto, no sorprende que facilite el acceso al crédito formal. En trabajos futuros se buscará descifrar por qué mujeres de estado civil casadas tienen mayor probabilidad de obtener un préstamo formal, mientras que hombres solteros de uno informal; por ahora, se presume que la popularidad de los créditos grupales, que frecuentemente se dedican exclusivamente a mujeres, podría ser una ruta para explicarlo. Poseer algún tipo de seguro posibilita otros servicios financieros, por lo que el resultado señala complementariedad en el acceso los formales.

Se identifica un segundo conjunto de variables con evidencia estadística de que influyen en las decisiones de crédito formal, pero no en las del informal: la posesión de activos, el número de integrantes del hogar, tener teléfonos celulares y contar con una cuenta de ahorro financiero. En general, un incremento en el valor de estas variables refleja mayor capacidad y disposición del individuo para pagar una deuda, determinantes para la evaluar el riesgo de incumplimiento en el sector formal. En el mercado informal las transacciones se realizan principalmente con familiares y amigos, quienes, al tener un conocimiento pleno de las características no observables del deudor, prestarán poca atención a las características observables. Finalmente, la edad parece no tener efecto estadístico en el sector formal, pero sí en el informal, donde la relación es positiva y decreciente. Si bien no es el propósito del presente descubrir las razones por las que la edad es relevante únicamente en un sector, el resultado advierte que estudios que vinculen el acceso a financiamiento con el ciclo de vida de los usuarios son necesarios para explicar el impacto agregado de las políticas de inclusión financiera.

El presente ensayo es un acercamiento inicial a las características de la demanda de crédito en México que permite derivar conclusiones acerca de factores que condicionan la eficacia de programas enfocados a fomentar la inclusión financiera en los mercados formales. No obstante, reconocemos sus limitaciones: problemas de autoselección en los distintos mercados crediticios, de causalidad simultánea y aquellos asociados al omitir factores no observables, por ejemplo, no han sido tratados debido a que la información disponible es de corte transversal. 


\section{ReFerencias}

Banco Mundial. (2018a). Inclusión Financiera Global. World Bank Group. Disponible en https://globalfindex. worldbank.org/\#data_sec_focus

Banco Mundial. (2018b). Indicadores de Desarrollo Mundial. World Bank Group. Disponible en https://data. worldbank.org/products/wdi

Banerjee, A., Duflo, E., Glennerster. R., \& Kinnan, C. (2015). The Miracle of microfinance? Evidence from a Randomized Evaluation. American Economic Journal: Applied Economics, 7(1), 22-53.

Besley, T. (1994). How do market failures justify interventions in rural credit markets? The World Bank Research Observer, 9(1), 27-48.

Conning, J., \& Udry, C. (2007). Rural financial markets in developing countries. In Robert Evenson \& Prabhu Pingali (eds.), Handbook of Agricultural Economics (Vol. 3, pp. 2857-2908).

Demigüç-Kunt, A., Beck, T., \& Honohan, P. (2008). Finance for All? Policies and Pitfalls in Expanding Access. A World Bank Policy Research Report. The World Bank.

Diagne, A. (1999). Determinants of household access to and participation in formal and informal credit markets in Malawi. FCND Discussion Paper 67. International Food Policy Research Institute.

ENIF (Encuesta Nacional de Inclusión Financiera). (2015). Instituto Nacional de Estadística y Geografía(INEGI). Base de datos en línea. Disponible en http://www.beta.inegi.org.mx/proyectos/enchogares/especiales/enif/2015/

Giné, X. (2011). Access to capital in rural thailand: An estimated model of formal vs. Informa3 Credit. Journal of Development Economics, 96(1), 16 -29.

Guirkinger C. (2008). Understanding the coexistence of formal and informal credit markets in Piura, Perú. World Development, 36(8), 1436-1452.

Gonzalez-Vega, C. (2003). Deepening rural financial markets: Macroeconomic, policy and political dimensions. International Conference on Paving the Way Forward for Rural Finance. Washington, D. C.: WOCCU \& USAID.

Jaffee, D., \& Stiglitz, J. E. (1990). Credit rationing. In Bejamin M. Friedman \& Frank H. Hahn (eds.), Handbook of Monetary Economics (Vol.2, pp. 838-888).

Levine, R. (1997). Financial development and economic growth: Views and agenda. Journal of Economic Literature, 35(2), 688-726.

Levine, R. (2005). Finance and growth: Theory and evidence. In Philippe Aghion y Steven N. Durlauf (eds.), Handbook of Economic Growth (Chap. 12., Vol. 1, pp. 865-934).

Levine, R., Loaiza, N., \& Beck, T. (2000). Financial intermediation and growth: Casualty and Causes. Journal of Monetary Economics, 46, 31-77.

Lloyd-Ellis, H., \& Berhardt, D. (2000). Enterprise, Inequality and Economic Development, Review of Economic Studies, 67(1), 147-168.

Maldonado, J. H., \& Gonzalez-Vega C. (2008). Impact of microfinance on schooling: Evidence from poor rural households in Bolivia. World Development, 36(11), 2440-2455.

McKenzie, D. J., \& Woodruff, C. (2008). Experimental evidence on returns to capital and access to finance in Mexico. The World Bank Economic Review, 22(3), 457-482.

Mohieldin, M. S., \& Wright P. W. (2000). Formal and informal credit markets in Egypt. Economic Development and Cultural Change, 48(3), 657-670. 